\title{
Lapicque's Investigations on the Chronaxie of Excitable Tissues.
}

\section{By John F. Fulton.}

$\mathrm{A}^{\mathrm{S}}$ there is no account in English, either in text$A$ book or journal, of the fundamental researches of Lapicque and his co-workers upon the physiology of nerve and muscle, any attempt to give such an account, however imperfect it be, carries with it its own justification. The need for a review of the work on chronaxie is the more pressing among nerve-muscle investigators, as many of Lapicque's most fundamental contributions, so often overlooked by English-speaking physiologists, lie scattered in the literature. The account which follows is taken from about sixty papers which have appeared largely in the Comptes rendus of the Paris Academy of Sciences (C.R. Acad. Sc.) and the Société de Biologie (C.R. Soc. Biol.) between 1908 and $x_{923}$. The nature of the article forbids a complete bibliography.

Many physiologists, especially Waller and Keith Lucas, have determined the total kinetic energy required to elicit a nerve impulse, and also the rapidity with which such an impulse springs into existence. In England the most fruitful results have been obtained by Lucas using a pendulum for the determination of absolute time of excitation. The use of the condenser discharge for this purpose, though at one time employed with great promise by Waller, has been developed with pre-eminent success by Lapicque. In the course of his investigations he has introduced certain new terms which require elucidation. "Rheobase" and "chronaxie" are the first which demand consideration.

In a paper (C.R. Soc. Biol., 67, 280) entitled "Définition expérimentale de l'excitabilité," "rhéobase" is defined as "l'intensité du courant constant à début brusque, à durée prolongée, qui donne le seuil [threshold] de l'excitation" (p. 283), and "chronaxie" as "la durée du passage du courant constant à début brusque, qui atteint le seuil de l'excitation avec une intensité égale au double de la rhéobase, soit pratiqué avec le voltage double du voltage rhéobasique." In other words, the rheobase is the intensity in volts of a constant current closed instantaneously which will just excite if continued indefinitely; while chronaxie is the time required for excitation by a current of intensity just double that of the rheobase. The rheobase may be very simply determined with a battery, a voltmeter, and an appropriate rheostat. The chronaxie, on the other hand, is determined, as already mentioned, by the discharge of a condenser, the procedure for which will be explained later.

Apparatus.-The apparatus devised by Lapicque, though in reality extremely simple, must be considered in some detail. For ordinary determinations of chronaxie on the nerves of frogs or mammals, a twoor four-volt accumulator is used as a source of current. This is led into an accurate potential reducer equipped with two hundred equal points of division (see Fig. I). Such réducteurs de potentiel may be obtained from G. Boulitte in Paris. ${ }^{1}$ The current is led from the potential reducer by way of a double-contact Morse

1 G. Boulitte, $x 5$ Rue Bobillot, Paris. Price, goo francs. key to a condenser of variable capacity, so arranged that the condenser is charged when the key is pressed down, and discharged when the key is released. The condenser ${ }^{2}$ contains a series of capacities ranging from 0.5 to 0 . a key) with this condenser is a less accurate condenser of a capacity of two microfarads. This is put into the circuit for the purpose of determining the rheobase, since its time of discharge is so long that it approximates to a constant current. In this way much laborious wiring is rendered unnecessary. It must be remembered that the wiring and mounting of this apparatus should be entirely non-inductive.

The condenser discharges (effected by the doublecontact key mentioned above) are led to the tissue through an ingeniously arranged resistance. The time required for the effective portion of a condenser discharge varies with the external resistance. Obviously the resistance of the nerve fibre or of the muscle substance of a frog varies enormously with the distance

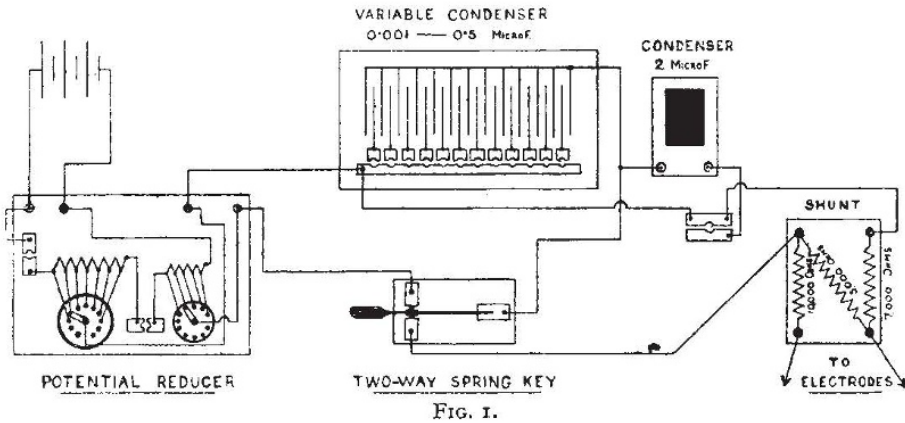

between the electrodes, the state of moisture of the preparation, size of fibre, etc., and it would be a hopeless task to redetermine a new resistance for each experiment. This difficulty has been overcome by interpolating a shunt of such low resistance (3000 ohms) as to make the resistance of the tissue excited much greater in comparison, and hence negligible. Other resistances are placed in series with the electrodes as shown in Fig. x. This resistance (in France la shunt de Lapicque), as well as the two-microfarad condenser and the non-polarisable electrodes about to be described, may also be obtained in Paris from Boulitte. The resistance is of graphite so as to have a very low temperature coefficient. When this is used the value $\mathrm{R}$ in the equation $\tau=\mathrm{CR} \times 0^{\circ} 37$ for the determination of the chronaxie in $\sigma$, may be taken as Io,000 ohms, and it is never altered for ordinary determinations. For further details on the external resistance Bourguignon's monograph (pp. 8o-82) must be consulted (Paris: Masson et Cie, I923).

As the rheobase determinations involve constant current of long duration, non-polarisable electrodes must be used. For the nerve a convenient small

${ }^{2}$ Much difficulty has been experienced in obtaining accurately constructed condensers of low and variable capacity at a reasonable price. structed condensers of low and variable capacity at a reasonable price. for $30 l .40 l$. I find also that Beaudouin, $3 \mathrm{I}$ Rue Lhomond, Paris, V, is willing to supply, for 700 to 800 francs, a modest but accurate design in two months from receipt of order. J. Carpentier (2o Rue Delambre) supplies, for $I 787$ francs, a more elaborate instrument of proper range of capacities in three months from receipt of order. 
hard-rubber chamber has been devised by Lapicque containing grooves for kaolin, upon which the nerve may be placed; other non-polarisable electrodes, provided they are not too large, serve quite as well. For the muscle, electrodes of thin silver wires, which have previously had deposited upon them by simple electrolysis a coating of silver chloride, are inserted directly into the muscle at convenient distances (0.5 cm. approx.). These may be made very simply by attaching the silver wire to the positive pole of a 4-volt accumulator and dipping it in Ringer solution for two minutes, the current being led away from the solution by a platinum wire. Such silver chloride electrodes must be renewed daily. Other more complex electrodes have been devised by Bourguignon (ז923) for stimulation through the skin.

Procedure.-To determine the chronaxie of the sciatic nerve of the frog, it is preferable to leave the parts so far as possible intact. On a pithed preparation, brain only or cord and brain, the uncut sciatic nerve is exposed and lifted with glass hooks on to the electrodes. Any excess kaolin or moisture between the electrodes is carefully removed and the preparation is allowed to remain quiescent preferably for a halfhour or longer.

Todetermine the rheobase the two-microfarad condenser is placed in the circuit with the variable condenser (at its highest capacity - I microfarad) and the least current necessary to excite is then found by gradually increasing the current in the réducteur de potentiel. The rheostat on the left of this instrument has twenty points; each of the twenty intervals may be further subdivided into ten by the rheostat on the right, thus giving 200 equal divisions of the potential. A voltmeter is ordinarily used to give the rheobase in absolute units, $0^{\circ} 2$ to $0^{\circ} 3$ volts being a reasonable value for the nerves of a frog, but it may be much higher. In a single experiment there is frequently considerable variation in the value of the rheobase, but this does not affect the value of the chronaxie. For more accurate determinations of rheobase and chronaxie, as in Bourguignon's ( 1923 ) experiments on man, the amperage is also controlled by an additional variable resistance.

To find the chronaxie, the rheobase just determined is doubled by means of the rheostat, or if it is preferred, by the voltmeter. However, with a Boulitte potential reducer it is always safe simply to double the reading of the rheostats. The two-microfarad condenser is withdrawn from the circuit, and the plugs of the variable condenser are arranged for a small capacity, usually 0.02 or 0.05 microfarads. The capacity is then gradually increased until a response is just visible, or if the muscle is attached to a writing lever, until the unloaded lever is just moved. This is the critical capacity from which the chronaxie is calculated by the formula: $\tau=\mathrm{CR} \times 0^{\circ} 37$. If the critical capacity proved $0^{\circ} \circ 82$ microfarads, and $\mathrm{R}$ as usual is ro,000 ohms, we have ( $\mathrm{C}$ being calculated in farads) :

$$
\begin{aligned}
& \tau=0.082 \times \frac{10,000}{I, 000,000} \times 0.37 \\
& \tau=\frac{0.082}{100} \times 0.37=0.000303 \text { or } 0.3 \sigma, \text { three ten- } \\
& \text { thousandths of a second, }
\end{aligned}
$$

which is normal for the sciatic nerve of the frog when not in connexion with the higher centres. The constant $0^{\circ} 37$ is purely empirical (C.R. Soc. Biol., 68, 797), and comes from a comparison of $\mathrm{CR}$ with the absolute time determined on the same preparation by means of a ballistic rheotome. For a discussion of this factor see Bourguignon's monograph (p. ro).

When the chronaxie of a muscle is determined, silver chloride non-polarisable electrodes are used as explained above. They are inserted just into the muscle substance separated by $0.5 \mathrm{~cm}$. or less, and the index of adequacy of stimulation is not the complete contraction of the muscle but rather the first visible twitch at the cathode. Otherwise there is a great spread of current and the chronaxie appears much larger than it is (Davis). Theoretically, the most satisfactory electrodes for determining the chronaxie of muscle are the sodium chloride pore electrodes used with success by Davis (J. Physiol., 57, lxxxi. 1923), which stimulate but a single muscle fibre (observed under the microscope).

Curarisation.-In the method just described for chronaxie determination in muscle, one might object quite justly that it is the chronaxie, not of the muscle fibres, but of the nerve fibres within the muscle which is being determined, and indeed Lapicque's contention that a skeletal muscle has normally the same chronaxie as its nerve has been criticised because of this technical difficulty. But such criticism comes from a failure to examine his evidence completely. It is admitted that the intra-muscular nerves are stimulated in this way; however, after curare has just caused a block for stimulation by the nerve, the chronaxie of the muscle is found to be double that of the nerve, and if the curare continues to act the chronaxie of the muscle continues to increase (C.R.Soc. Biol., 68, 1007). If, on the other hand, the curare-effect is brought about by strong strychnine ( $\mathrm{I}$ in $\mathrm{I}$ to 2000 ) applied peripherally, the chronaxie of the nerve trunk, when the block is just effective, is found to have sunk to $0^{\circ} 5^{\sigma}$ or less, while that of the muscle remains " normal," i.e. at $0^{\prime} 3^{\sigma}$ or thereabouts, which is the chronaxie previously possessed by the nerve $(C . R$. Soc. Biol., 74, ror2). From this the conclusion is drawn that normally there exists a condition of iso-chronism between a motor nerve and a voluntary muscle which it innervates, and that curarisation is brought about when the time relations are disrupted to such an extent that the chronaxie of one differs from the other by a hundred per cent.; in other words, heterochronism results in curarisation.

There are four possible ways in which curarisation might be brought about (C.R. Soc. Biol., 72, 283) :

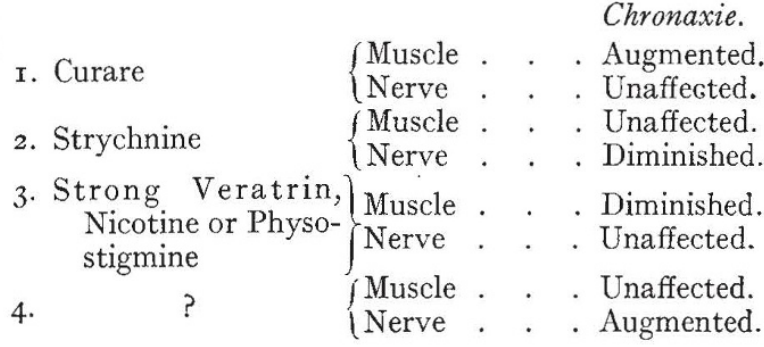

It will be seen that only three methods for producing curarisation are realised practically, as there is no way as yet known for increasing the chronaxie of the nerve 
without at the same time enhancing that of the muscle. Hypertonic saline augments the chronaxie of both (Laugier).

It follows from Lapicque's theory that a drug such as strychnine, which produces a block by diminishing the chronaxie of the nerve, should be counteracted in its action by a drug which diminishes that of the muscle. Experimentally it is found that veratrin, nicotine, and physostigmine restore a nerve muscle preparation curarised by strychnine (C.R. Soc. Biol., 74, IOI2). Curare itself is likewise antagonised by physostigmine (C.R. Soc. Biol., 72, 674) and by nicotine (Langley; also Lapicque, J. Physiol. Path. gén., 20,488 ).

In all of the investigations on chronaxie, no evidence has been found in muscle for the existence of KeithLucas's $\beta$-substance, and it has been sought for by means of rectangular rheotome currents of short durations, such as Lucas himself used, as well as with condensers, yet in every case with strength-duration curves the points have been continuous. Either, therefore, Lucas was in error, perhaps as a result of using large non-polarisable electrodes on excised muscle (Davis), or alternatively the condenser discharge may give only the chronaxie of the $\beta$-substance without exciting the substance of the muscle itself; perhaps in the muscle there exists another curve altogether, such as one finds in muscle in which the nerve has degenerated; but this is unlikely, and one cannot as yet decide. Lapicque believes, in view of the theory of isochronism, that the action current of the nerve excites the muscle directly, without the intermediation of a junctional tissue, a view strongly upheld by Forbes $(A m$.$) .$ Physiol., 66, 553-6r7).

Chronaxie and the Morphological Structure of the Nerve Fibre.--It is not surprising that in the same animal there is wide variation in the chronaxie of different nerves. In general, the nerves to the rapidly contracting muscles have the smallest chronaxie. But a fact much more significant than this comes from a study of the histological structure of nerve fibres in relation to their respective chronaxies. If, in the frog, the average diameter of the fibres in various nerve trunks is tabulated beside their chronaxies, we see that the chronaxie decreases with an increase in diameter

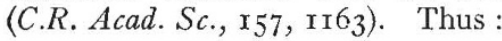

\begin{tabular}{|c|c|c|c|c|}
\hline Nerve and Muscle. & & $\begin{array}{c}\text { Chronaxie } \\
\text { (in } \sigma) .\end{array}$ & $\begin{array}{c}\text { Average } \\
\text { Diameter } \\
\text { (in } \mu \text { ). }\end{array}$ & $\begin{array}{c}\text { Product : } \\
\text { Chron. } \times \text { Diam. }\end{array}$ \\
\hline Sciatic-gastrocnemius & . & 0.3 & I $8-23$ & $97-\mathbf{I} 6 \mathbf{r}$ \\
\hline Brachial . & . & 0.6 & $12-13$ & 86-ror \\
\hline Sartorius i & $\therefore$ & $I \cdot O$ & IO-II & IOO-I10 \\
\hline Vagus (cardiac branch) & $:$ & $2 \cdot 0$ & 7 & 98 \\
\hline Nerve to stomach & - & $20 \cdot 0$ & 2 & 80 \\
\hline
\end{tabular}

The product of the chronaxie and the square of the diameter gives a value which is very nearly constant, a fact the meaning of which is as yet unknown. It is interesting that stretching a frog's nerve by a weight of ten grams, which would tend to diminish the diameter, causes an augmentation of the chronaxie (C.R. Soc. Biol., 69, 46). Further evidence comes from the rabbit, where one finds that the white rapid muscles, such as the adductor magnus and gastrocnemius, are innervated by fibres $I_{3} \mu$ in diameter, while the slow red muscles, soleus and semitendinosus, possess nerve fibres but $8 \mu$ in average cross-section.

Of even greater importance, however, are the morphological changes shown to occur in nerve fibres when under the influence of anæsthetics, decalcificants, certain alkaloids, and extreme cold. Lapicque and Legendre, in a class demonstration of chronaxie determination, observed in the sciatic nerve of a frog which previously had been exposed to extreme cold, an extraordinarily short chronaxie ${ }^{3}$ with a high rheobase. Microscopic examination of the nerve revealed the fact that the myelin sheaths were greatly swollen, in some cases almost to the obliteration of the axis cylinder. It happened that at the time Lapicque was himself studying the action of chloroform and the oxalates, which also produced in nerves abnormally small chronaxies associated with extremely high rheobases, the latter increasing to complete inexcitability in the case of chloroform and ether.

Microscopic observation of nerves subjected to the action of such reagents reveals the fact that, pari passu with the increasing rheobase and diminution of the chronaxie, there appear swellings of the myelin sheaths such as occur also after extreme cold. This interesting observation led to a perfecting of the techniques for examining the living nerve fibre under the influence of anæsthetics. Lapicque and Legendre ( $J$. Physiol. Path. gen., 21, I63, I922) have found that the nerve must be observed without teasing out the separate fibres; for this purpose the peroneal or tibial of a pithed frog is dissected out carefully, and everything is cut away between the knee and the ankle except the nerve. To ensure that the nerve is in every way normal the sciatic trunk is stimulated in the thigh to see whether the digits move. A cover slip, the corners of which have been turned down with heat, is put over the nerve. The preparation, pinned to a cork stage with a glass window, is now made ready for observation with an oil-immersion objective. With blotting-paper various reagents may be sucked through under the raised cover slip. By this procedure Lapicque and Legendre have shown that as the threshold for stimulation gradually rises to complete inexcitability under the influence of ether, chloroform, novocaine and cocaine, there appears first a translucency of the myelin, followed by swelling of this sheath and the formation of protuberances, especially at the nodes. (Cf. the beautiful photomicrographs in Lapicque and Legendre's paper, I922.) The condition is reversible, restoration taking place in normal saline. It follows from this that the "decrement" of the Cambridge School is accompanied by a morphological lesion of the nerve trunk.

'The swelling of the myelin which occurs with ether and chloroform is believed by Lapicque to be due to the absorption of water. Mme. Lapicque has pointed out that the more rapid the chronaxie of a given muscle the more will be its imbibition when placed in hypotonic saline. In hypertonic saline the chronaxie of a nerve is augmented, owing presumably to the extraction of water from the sheath (Lapicque et Lapicque, r92I). Also curare, which causes the chronaxie of a muscle to rise, diminishes its imbibition.

In a short review such as this, which aims primarily

${ }^{3}$ Normally, however, the chronaxie increases when the temperature is lowered moderately, as one would expect.

NO. 2838 , vOL. I I 3$]$ 
at describing the apparatus and procedure for chronaxie determination, one cannot treat in detail the results which have thus far been obtained. One might mention the beautiful experiments with repetitive stimulation of known intensity, duration, and interval, which have been employed with such success by the Chauchards in analysing the action of the chorda tympani and the vagus, and by Koenig in his investigations on the excitability of the vaso-motor and "pigmento-motor" nerves. These investigations have shown that a difference in chronaxie between a nerve and its effector is overcome by repetitive stimulation. Bourguignon in his monumental work on chronaxie in man has shown that the extensors have in general one chronaxie $(0 \cdot 10-0 \cdot 16 \sigma)$ while the flexors have another $(0.44-0.72 \sigma)$.

Lapicque has refrained as yet from any detailed consideration of the application of the work on chronaxie to the theories of the functional activity of the central nervous system. He has, however, made several suggestions (J. Psychol. norm. path., 8, I), one of which (Rev. gén. Sci., 2 I, I03) must be mentioned. With double condensers the ascent of the discharge, instead of being instantaneous as with a single condenser, is gradual, not unlike a sine curve. If, therefore, two sciatic-gastrocnemius preparations, one from a toad (chronaxie $x \cdot 3^{\sigma}$ ), the other from a frog (chronaxie $0.3 \sigma$ ), are placed side by side across the same pair of electrodes, each receiving in consequence the entire effect of the discharge, when the condensers are charged at a small capacity, only the frog's muscle will contract, and only the toad's at a higher capacity. This is the effect when the intensity is small; if now the intensity of the discharge is increased, both muscles will contract simultaneously at either capacity. "Cette expérience est pour moi une image de la façon dont pourrait fonctionner un centre nerveux quand il $y$ a quatre neurones, deux centripètes et deux centrifuges" (p. I I6).

Another fact of singular importance, which must be taken into consideration in enunciating a theory of central action based on chronaxie, is the influence which the higher centres (the region in front of the optic lobes in the frog) have on the chronaxie of the sciatic nerve (M. Lapicque, C.R. Soc. Biol., 88, 46, 1923). In a normal frog when these centres are intact the chronaxie may be as small as 0.19 or $0.2 \sigma$, but immediately the brain is sectioned behind the optic lobes the chronaxie rises as high in some experiments as 0.4 or 0.42 . Usually the effect of cutting off the higher centres, or of cutting the nerve itself, is to double the chronaxie. This obviously represents a type of nervous influence which, wholly unlike the propagated disturbance, has never before been described. It is akin to a polarisation which, if it passes via the pyramidal tracts, must be able to exert its effect across synapses. Perhaps this accounts for the greater facility with which reflexes are elicited in the spinal preparation than in the decerebrate preparation.

I should like to take this opportunity of expressing my most sincere thanks to Prof. and Mme. Lapicque and to Dr. Laugier for their great kindness, especially in granting me the facilities of their laboratory at the Sorbonne in January 1924 .

\section{The Memorial to Lord Lister.}

IMMEDIATELY after the death of Lord Lister in I9I2, the question of a memorial to him was discussed by fellows of the Royal Society and of the Royal Colleges of Physicians and Surgeons. Some delay, necessarily occurred before action could be taken, but eventually a provisional committee consisting of representatives of the Royal Society and the Royal College of Surgeons met and agreed to issue, not only to men distinguished in science and surgery, but also to men of eminence in public life and in various branches of knowledge, both at home and abroad, a request that they would consent to form themselves into a general committee for the purpose of considering the question of a suitable memorial and taking such steps as they. might think fit to organise the collection of subscriptions. This invitation met with a ready response and a large and influential committee consisting of foreign, ambassadors and ministers, representatives of universities, scientific and learned societies, city companies, and men eminent in every branch of knowledge was constituted.

An executive committee, with Sir Archibald Geikie, then president of the Royal Society, as chairman, Viscount Hambleden as vice-chairman, Lord Rothschild and Sir W. Watson Cheyne, treasurers, and Sir John Rose Bradford, honorary secretary, was appointed. This committee considered several proposals for a memorial and came to the conclusion that the most suitable would be one comprising :
I. A tablet with medallion and inscription to be placed in Westminster Abbey.

2. The establishment of an International Lister Memorial Fund for the advancement of surgery, from which either grants in aid of researches bearing on surgery, or awards in recognition of distinguished contributions to surgical science should be made, irrespective of nationality.

3. A monument to be erected in a public place in London.

This proposal was put forward at a public meeting held at the Mansion House and unanimously adopted. Subscriptions were received not only from all parts of the Empire, but also from Buenos Aires, Denmark, France, Germany, Greece, Holland, Hungary, Italy, Japan, Portugal, Sweden, Switzerland, and the United States. The Glasgow Lister Memorial Committee also contributed a substantial sum. The work of the committee was to a great extent held in abeyance during the War, but a memorial tablet was unveiled in the north aisle of Westminster Abbey in November I9I5 without public ceremony. The unexpended balance of subscriptions received was invested, eventually increasing the sum collected for the memorial by $2600 l$., making a total of nearly $12,000 l$. Towards the end of $1920,5000 l$. trustee stock was handed over to the Royal College of Surgeons of England to provide a fund from which every three years a sum of $500 l$. is to be awarded, together with a bronze medal, in recogni-

$$
\text { NO. } 2838 \text {, VOL. I I } 3 \text { ] }
$$

